\title{
Focus on tracheostomy
}

Tracheostomy has long been a vital standard of the otolaryngologist's repertoire. An important review article in this issue by the National Confidential Enquiry into Patient Outcome and Death ('NCEPOD') in the UK examines current practice in tracheostomy. ${ }^{1}$ The emergence of percutaneous tracheostomy techniques used by critical care doctors has changed the scope of this operation, with the majority (69.6 per cent) of procedures being undertaken in this way in the UK during the survey period. Surgical tracheostomy continues to be undertaken on those patients unsuitable for percutaneous techniques. Patients undergoing surgical tracheostomy tend to have unfavourable anatomy, which has implications for training. ${ }^{2}$ With the procedure becoming more commonplace, there could be a tendency to underplay the potential complications. The review found that complications were common, both in the critical care and ward environment, occurring in just over 20 per cent of cases. Accidental decannulation and hypoxia were the most frequent serious complications. Another issue is the increasing number of patients that cannot be successfully decannulated, with discharge to a community setting becoming difficult.

A previous Editorial in The Journal of Laryngology \& Otology demonstrated the utility of The Journal Archives in documenting the history of Ménière's disease. $^{3}$ An article in the current issue adds to archive material in reporting the effects of intratympanic methylprednisolone perfusion in patients with intractable Ménière's disease. ${ }^{4}$ She and colleagues report effective control of vertigo and improved functional activity in this difficult group of patients. This method of treatment appears also to offer the advantage of hearing preservation compared with the potentially ototoxic effects of intratympanic aminoglycoside treatment. ${ }^{5}$ The increasing awareness of the use of intratympanic medication has been one of the most exciting developments in otology in recent years. ${ }^{6,7}$

Finally, perhaps the most striking image in this issue of The Journal is of a green temporal bone in a patient who had undergone long-term treatment with tetracycline antibiotics. ${ }^{8}$ Antibiotics are now used for long periods in the treatment of many conditions, including rhinosinusitis. This article serves as a reminder of the potential hazards of this type of treatment.

\section{ROBIN YOUNGS EDWARD FISHER} Senior Editors

References

1 Wilkinson KA, Freeth H, Martin IC. Are we 'on the right trach?' The National Confidential Enquiry into Patient Outcome and Death examines tracheostomy care. J Laryngol Otol 2015;129: 212-6

2 Steele PR, Mountain RE. Elasticated retractors in tracheostomy. J Laryngol Otol 2013;127:1141-2

3 Flood LM, Kenyon G. Concepts of Ménière's disease in the Archives of The Journal of Laryngology and Otology. $J$ Laryngol Otol 2014;128:308-17

4 She W, Lv L, Du X, Li H, Dai Y, Lu L et al. Long-term effects of intratympanic methylprednisolone perfusion treatment on intractable Ménière's disease. J Laryngol Otol 2015;129:232-7

5 Wasson J, Upile N, Pfleiderer A. Intratympanic gentamicin treatment for unilateral Ménière's disease: long-term follow up of a proven regime. J Laryngol Otol 2013;127:20-4

6 Belhassen S, Saliba I. Intratympanic steroid injection as a salvage treatment for sudden sensorineural hearing loss. J Laryngol Otol 2014;128:1044-9

7 Sozen E, Erol SB, Yildirim O, Coskun BU, Basak T, Kayhan FT et al. Local and systemic effects of low-dose transtympanic methotrexate: in vivo animal study. J Laryngol Otol 2013;127: $148-52$

8 Farahnik B, Zaghi S, Hendizadeh L, Gopen Q. Rusty green stained temporal bone associated with exposure to tetracycline: an unusual presentation of black bone disease. J Laryngol Otol 2015;129:276-8 